University of New Hampshire

University of New Hampshire Scholars' Repository

Physics Scholarship

Physics

\title{
4-1994
}

\section{Observations of Cygnus X-1 COMPTEL during 1991}

\author{
Mark L. McConnell \\ University of New Hampshire - Main Campus, mark.mcconnell@unh.edu \\ D J. Forrest \\ University of New Hampshire - Main Campus \\ James M. Ryan \\ University of New Hampshire, James.Ryan@unh.edu \\ W Collmar \\ Max-Planck-Institut für extraterrestriche Physik \\ V. Schonfelder \\ Max-Planck-Institut für extraterrestriche Physik
}

See next page for additional authors

Follow this and additional works at: https://scholars.unh.edu/physics_facpub

Part of the Astrophysics and Astronomy Commons

\section{Recommended Citation}

M. McConnell, D. Forrest, J. Ryan, W. Collmar, V. Schoenfelder, H. Steinle, A. Strong, R. van Dijk, W. Hermsen, and K. Bennett, 'Observations of Cygnus X-1 COMPTEL during 1991', The Astrophysical Journal, vol. 424, p. 933, Mar. 1994.

This Article is brought to you for free and open access by the Physics at University of New Hampshire Scholars' Repository. It has been accepted for inclusion in Physics Scholarship by an authorized administrator of University of New Hampshire Scholars' Repository. For more information, please contact Scholarly.Communication@unh.edu. 


\section{Authors}

Mark L. McConnell, D J. Forrest, James M. Ryan, W Collmar, V. Schonfelder, H Steinle, A W. Strong, R Van Dijk, W Hermsen, and K Bennett 
THE Astrophysical Journal, 424:933-939, 1994 April 1

(1) 1994. The American Astronomical Society. All rights reserved. Printed in U.S.A.

\title{
OBSERVATIONS OF CYGNUS X-1 BY COMPTEL DURING 1991
}

\author{
M. MCCONnell, ${ }^{1}$ D. Forrest, ${ }^{2}$ AND J. Ryan ${ }^{3}$ \\ Space Science Center, University of New Hampshire, Durham, NH 03824 \\ W. Collmar, ${ }^{4}$ V. Schönfelder, ${ }^{5}$ H. Steinle, ${ }^{6}$ and A. Strong ${ }^{7}$ \\ Max Planck Institute for Extraterrestrial Physics, Garching, Germany \\ R. VAN DIJK ${ }^{8}$ AND W. HERMSEN ${ }^{9}$ \\ Laboratory for Space Research (ROL), Leiden, The Netherlands \\ AND \\ K. BENNETT ${ }^{10}$ \\ Astrophysics Division, ESTEC, Noordwijk, The Netherlands \\ Received 1993 August 16; accepted 1993 October 7
}

\begin{abstract}
The COMPTEL experiment on the Compton Gamma-Ray Observatory (CGRO) has observed the Cygnus region on two occasions during the first (sky survey) phase of its mission. These data represent the most sensitive observations to date of Cygnus X-1 in the $0.75-30 \mathrm{MeV}$ range. The observations in 1991 June and August both showed evidence for emission in the $0.75-2 \mathrm{MeV}$ energy range. The flux level was larger by about a factor of 2 during the August observation. The spectral data were analyzed in the context of a Wien spectral model (the high-energy limit of the Sunyaev-Titarchuk Comptonization spectrum). Fits to this model gave electron plasma temperature $(k T)$ values of $192 \pm 27 \mathrm{keV}$ and $204 \pm 21 \mathrm{keV}$ for the June and August data, respectively. These values are much higher than those typically derived from hard X-ray data, suggesting that an alternative to the single-temperature Comptonization model may be required to explain the entire spectrum. The present data may be explained by resolving the inadequacies of the standard Comptonization model which is used to fit the X-ray data and/or by incorporating a spectral component which represents the reflection of hard X-rays from an optically thick accretion disk. Finally, there is no evidence in these data for any substantial hardening of the spectrum above $1 \mathrm{MeV}$ (the so-called " $\mathrm{MeV}$ excess"), such as that suggested in the past by several balloon and satellite observations.

Subject headings: black hole physics - gamma rays: observations - stars: individual (Cygnus X-1) - X-rays: stars
\end{abstract}

\section{INTRODUCTION}

Cygnus $\mathrm{X}-1$ was first detected as an X-ray source almost 30 years ago. The present picture of Cygnus $X-1$ is that of a binary system in which a compact object orbits around the O-type supergiant HD 226868 with a period of 5.6 days (Gies \& Bolton 1986). The most recent dynamical evidence suggests that the unseen companion has a mass of $6 M_{\odot}$ (Dolan \& Tapia 1989; Dolan 1992). Since most theories predict a maximum neutron star mass approaching $3 M_{\odot}$, the compact companion is generally considered to be a black hole. Mass lost by the primary through stellar winds accretes onto the black hole, leading to an appreciable emission of thermal $\mathrm{X}$-rays. At hard X-ray energies, Cygnus $\mathrm{X}-1$ is one of the brightest sources in the sky. As a steady X-ray source, it is rivaled only by the $\mathrm{Crab}$ in terms of its apparent luminosity.

The X-ray emission from Cygnus X-1 is known to be highly variable on a wide range of time scales (from milliseconds to

\footnotetext{
${ }^{1}$ E-mail:mmcconnell@unh.edu

2 E-mail: forrest@unhsmm.unh.edu

${ }^{3}$ E-mail:jryan@unh.edu

4 E-mail:wec@ibma.ipp-garching.mpg.de

${ }^{5}$ E-mail:vos@ibma.ipp-garching.mpg.de

6 E-mail: hcs@ibma.ipp-garching.mpg.de

7 E-mail: aws@ibma.ipp-garching.mpg.de

8 Also Astronomical Institute “Anton Pannekoek," University of Amsterdam.E-mail: vandijk@rulrol.leidenuniv.nl

${ }^{9}$ E-mail: hermsen@rulrol.leidenuniv.nl

10 E-mail:kbennett@estsa2.estec.esa.nl
}

months). The early data from Cygnus $\mathrm{X}-1$ indicated that the soft X-ray emission $(E<10 \mathrm{keV})$ was found at one of two levels, with the hard X-ray emission $(\sim 100 \mathrm{keV})$ anticorrelated with the soft X-ray flux. These two levels were termed the "high" and "low" X-ray states, based on the flux level of the soft X-rays. Cygnus X-1 seems to spend most $(>80 \%)$ of its time in the "low" state. In a similar fashion, Ling et al. (1987) have defined three different levels for the hard X-ray flux. In order of increasing flux, these are termed the $\gamma_{1}, \gamma_{2}, \gamma_{3}$ states. Based on data from $H E A O$ 3, there appears to be no direct correlation between the soft and hard X-ray fluxes.

Several authors (e.g., White, Fabian, \& Mushotzky 1984; Owens \& McConnell 1992) have discussed the similarities between the X-ray spectra of galactic black hole candidates and the X-ray spectra of AGNs. Such comparisons are not surprising, given that, in both cases, the emission is believed to result from accretion onto a black hole. These similarities are especially apparent at soft X-ray energies, where common features include an iron $\mathrm{K} \alpha$ emission feature near $6.2 \mathrm{keV}$, a soft blackbody component with a temperature $\sim 0.25 \mathrm{keV}$, and a hard power-law component (Barr, White, \& Page 1985; Pounds et al. 1990; Balucinska \& Hasinger 1991). In AGNs, these features have been incorporated into models involving the reprocessing of radiation by reflection off cold matter in the vicinity of the accretion disk (e.g., Lightman \& White 1988; Pounds et al. 1990; Matt, Perola, \& Piro 1991; Ross, Fabian, \& Mineshige 1992; Ross \& Fabian 1993). 
The hard X-ray spectrum of Cygnus X-1 at energies up to several hundred $\mathrm{keV}$ has traditionally been modeled as an inverse Compton spectrum, whereby the hard X-rays result from the scattering of hot electrons off ambient UV photons (Sunyaev \& Titarchuk 1980). Much of the available hard X-ray data fit this model quite well and indicate an electron temperature of $\sim 27-80 \mathrm{keV}$ and an optical depth $(\tau)$ of $\sim 1-5$. More recent work (Done et al. 1992; Haardt et al. 1993) suggest that an additional spectral component must also be considered in this energy range, namely, the reflection of the hard X-rays (generated in an optically thin accretion disk corona) off the (optically thick) accretion disk. This model suggests both a higher electron temperature $(k T \sim 140-200 \mathrm{keV})$ and a smaller optical depth $(\tau \sim 0.3)$ than previously considered for the energetic electron population.

Some of the more intriguing measurements of Cygnus X-1 are those which indicate a hardening of the spectrum in the region around $1 \mathrm{MeV}$ (Baker et al. 1973; Mandrou et al. 1978; Ling et al. 1987; Bassani et al. 1989; McConnell et al. 1989). The reported levels of emission are much higher than that which would be expected based on a simple extrapolation of the hard X-ray data. These data suggest the possibility of an additional component to the high-energy spectrum. Most notable are the observations by $H E A O 3$ of a time-variable feature near $1 \mathrm{MeV}$ (Ling et al. 1987), which has been explained in the context of a hot electron-positron plasma $(k T \sim 400$ $\mathrm{keV}$ ) in the inner region of the accretion disk (Liang \& Dermer 1988; Liang 1990). The HEAO 3 data suggest that the excess $\mathrm{MeV}$ emission may be associated with the $\gamma_{1}$ hard X-ray state. However, such an association is not apparent in other observations of excess emission (Owens \& McConnell 1992). The OSSE experiment has recently observed Cygnus X-1 in its $\gamma_{1}$ state and placed upper limits on the $\mathrm{MeV}$ emission which are well below the flux levels observed by HEAO 3 (Leising et al. 1993). These data suggest that there is no direct correlation between the hard X-ray flux and the MeV flux.

Although the evidence for emission at energies $\sim 1 \mathrm{MeV}$ is limited, it does suggest some very interesting physics. Emission at energies greater than a few $\mathrm{MeV}$ (such as that reported by McConnell et al. 1989) may not be possible from a thermal electron population (e.g., Dermer \& Liang 1989). Any emission greater than a few MeV may therefore be a result of either some thermal ion population or some nonthermal process. For example, some black hole accretion disk models postulate the development of a two-temperature plasma (e.g., Shapiro, Lightman, \& Eardley 1976) in which the ion population reaches a much higher temperature $\left(T_{i} \sim 10^{12} \mathrm{~K}\right)$ than the electron population $\left(T_{e} \sim 10^{8} \mathrm{~K}\right)$. In this model, pion production may take place within the thermalized ion plasma, giving rise to a spectral signature in this part of the spectrum (e.g., Dahlbacka, Chapline, \& Weaver 1974; Kolykhalov \& Sunyaev 1979; Eilek 1980; Giovannelli, Karakula, \& Tkaczyk 1982; Eilek \& Kafatos 1983). A two-temperature accretion flow in the vicinity of a black hole might also be sufficiently hot to induce nuclear excitations in the accreting plasma (Higdon \& Lingenfelter 1977). Unfortunately, the line flux in such a situation would be limited by nuclear breakup reactions (Aharonian \& Sunyaev 1984; Guessom \& Dermer 1988). However, neutrons generated by reactions within the accretion flow could escape and interact in the companion star, thus producing line emission at $2.2 \mathrm{MeV}$ (e.g., Guessom \& Dermer 1988; Guessom 1989). Hence, emission at these energies might be considered as a useful probe of a two-temperature accretion disk. The prospect of nonthermal emission (i.e., particle beaming) offers several additional explanations for emission at energies greater than $1 \mathrm{MeV}$. For example, Bednarek et al. (1990) have offered one possible explanation for the data of McConnell et al. (1989) as resulting from a beam of electrons interacting in the atmosphere of the companion star.

In addition to the various reports of excess emission in the region near $1 \mathrm{MeV}$, there are also several observations which have found no evidence for such emission. The Compton telescope observations of both MPE (Schönfelder \& Lichti 1974; Varendorff et al. 1990) and UCR (White et al. 1980) have placed upper limits on the emission which are well below the level of the observed excess. Taken collectively, the entire body of observational evidence suggests that any "MeV excess" must be time variable.

The COMPTEL experiment on the Compton Gamma-Ray Observatory is designed to measure gamma-radiation in the energy range from 0.75 to $30 \mathrm{MeV}$ (Schönfelder et al. 1993). The unprecedented sensitivity of COMPTEL permits a detailed study of the Cygnus X-1 emission in this portion of the spectrum. During the first phase of the CGRO mission (the 18 month sky survey period), Cygnus X-1 was within the field of view of COMPTEL on two separate occasions.

\section{INSTRUMENTATION AND OBSERVATIONS}

The COMPTEL experiment is capable of imaging $\gamma$ radiation within a field of view of $\sim 1$ sr. It consists of two independent layers of detection modules separated by $150 \mathrm{~cm}$. The upper (D1) layer is composed of seven independent NE213A liquid scintillator detectors, each $28 \mathrm{~cm}$ in diameter and $8.5 \mathrm{~cm}$ thick. The lower (D2) layer is composed of 14 independent $\mathrm{NaI}(\mathrm{Tl})$ detectors, each $28 \mathrm{~cm}$ in diameter and 7.5 $\mathrm{cm}$ thick. An event is defined as a coincident interaction in a single D1 detector and a single D2 detector. For each event, the total energy is estimated as the sum of the energy losses in both D1 and D2. The interaction locations in both detectors are determined (with a spatial location uncertainty of $\sim 2.0 \mathrm{~cm}$ ) using the relative responses of the various PMTs which are affixed to each D1/D2 detector. The measurement of the time of flight (TOF) between D1 and D2 and the pulse shape (PSD) in $\mathrm{D} 1$ allow for the rejection of a large fraction of background events, including both upward-moving and neutron-induced events. A more detailed description of COMPTEL can be found in Schönfelder et al. (1993).

The basic principle of COMPTEL imaging is governed by Compton scattering. If an event is completely absorbed by the interactions in D1 and D2, then we have an accurate measure of both the scattered electron energy and the scattered photon energy. Knowledge about the interaction locations in both the upper (D1) and lower (D2) detector layers also provides information about the path of the scattered photon. Without more complete knowledge regarding the direction of the scattered electron, the possible arrival direction of that event is confined to a circle on the sky. This event circle is defined to have an angular radius $(\bar{\phi})$ determined by the Compton scattering formula:

$$
\cos \bar{\phi}=1-\frac{m_{e} c^{2}}{E_{2}}+\frac{m_{e} c^{2}}{E_{1}+E_{2}},
$$

where $m_{e} c^{2}$ is the electron rest mass energy and $E_{1}$ and $E_{2}$ are the energy deposits measured in D1 and D2, respectively. The superposition of many event circles can lead to the localization 
of a source by determining the direction in which the majority of the event circles intersect. This can most easily be seen in cases where there is a high signal-to-noise ratio (Winkler et al. 1992). The event circles represent the basic principle of Compton telescope imaging. In practice, the analysis of COMPTEL data is far more complex. Many instrumental effects complicate this simplified approach. For example, incomplete energy absorption in either D1 or D2 can render equation (1) invalid; effects such as these are taken into account in the final analysis (see $\S 3$ ).

The initial viewing plan for the sky-survey phase of CGRO included a single 2 week observation of the Cygnus region. This observation (viewing period 2.0) began, as scheduled, on 1991 May 30. After only 9 days, the observation was interrupted due to the declaration of a solar target of opportunity for CGRO. A second (unexpected) opportunity to observe the Cygnus region came less than 2 months later, when another target-of-opportunity was declared to observe Cygnus X-3. This 7 day target-of-opportunity (viewing period 7.0), which was prompted by an intense outburst of radio emission from Cygnus X-3, began on 1991 August 8. In each case, Cygnus X-1 was well-placed for observation within the COMPTEL fieldof-view. During these observations COMPTEL was operating at only $\sim 80 \%$ of its total efficiency, a result of having several individual modules turned off in order to minimize the effects of continued outgassing. A summary of both observation periods is provided in Table 1 .

\section{DATA ANALYSIS}

The analysis of COMPTEL data is performed within a three-dimensional data space which is defined by the quantities $(\chi, \psi, \bar{\phi})$. The angles $(\chi, \psi)$ define the direction of the scattered photon (i.e., the vector defined by the interaction locations in D1 and D2) as projected backward onto the sky. These angles can be defined in any convenient coordinate system; the present analysis equates $(\chi, \psi)$ with galactic coordinates $(l, b)$. The third dimensions of this dataspace $(\bar{\phi})$ is the Compton scatter angle, as computed from equation (1). For a point source, the distribution of events in this data space is generally contained within the interior of a cone whose apex corresponds to the direction of the source. This distribution corresponds to the point spread function (PSF) of COMPTEL.

The ability to generate reliable PSFs is vital to the successful analysis of COMPTEL data. We have developed three independent procedures for generating PSFs. The first approach relies on preflight calibration data from COMPTEL to empirically generate a PSF; this approach incorporates all of the detector physics, but is limited by statistics, by background rejection and by effects related to the finite distance of the calibration source. The second method is based on separate preflight calibrations of both the individual D1 and D2 detectors; this approach depends on accurate knowledge and subsequent parameterization of several physical processes. Finally,

TABLE 1

Comptel Phase 1 Observations of CYGNUS X-1

\begin{tabular}{llc}
\hline \hline Observation & \multicolumn{1}{c}{$\begin{array}{c}\text { Dates } \\
(1991)\end{array}$} & $\begin{array}{c}\text { Angle } \\
\text { from Axis }\end{array}$ \\
\hline $2.0 \ldots \ldots \ldots$ & May 30-Jun 8 & 2.0 \\
$7.0 \ldots \ldots \ldots$ & Aug 8-15 & 11.4 \\
\hline
\end{tabular}

Monte Carlo simulations (based on the CERN GEANT code) are also used to generate PSFs. The use of three independent techniques permits detailed cross checks and leads to increased confidence in our knowledge of the PSF. All three approaches give reasonably good agreement, although several minor differences are still being investigated. The current uncertainties in the PSF are estimated to contribute a systematic error of not more than $\sim 0.5-1.0$ to the present source location capability of COMPTEL, based on the analysis of events from some known sources. The present analysis relies on simulated PSFs, which are currently considered to be the most reliable.

The PSF is a convolution of monoenergetic instrument responses and as such depends not only on the source location within the field of view, but also on the assumed emission spectrum of the source. In the study of Cygnus X-1, we generally employed PSFs based on a Wien spectrum, which represents the high-energy limit of the Sunyaev-Titarchuk inverse-Compton spectrum (Sunyaev \& Titarchuk 1980). The form of this spectrum is given by

$$
\frac{d N}{d E}=A E^{2} e^{-E / k T},
$$

where $A$ is some normalization constant, $E$ is the photon energy and $k T$ is the electron plasma temperature. Note that, in addition to the normalization, the Wien spectrum has only a single parameter: the electron temperature $(k T)$ of the plasma which provides the scattering medium. Given the high-energy threshold of $\sim 750 \mathrm{keV}$, the COMPTEL observations are relatively insensitive to the Compton scattering optical depth (at least for the range of electron temperatures considered here).

In order to maximize the signal-to-noise, various event selections are imposed on the analysis. These include the following: (1) restrictions on D1 pulse shape (PSD) to select only those events consistent with incident photons; (2) a TOF selection to reject all but "forward" scattered photon events; (3) a scatter angle $(\bar{\phi})$ selection to restrict the analysis to a range of values which is dominated by source events rather than by background events; and (4) a selection is made so that any event whose event circle passes within $5^{\circ}$ of Earth's disk is rejected (thus minimizing the background of Earth's albedo $\gamma$-rays).

The derivation of an image from COMPTEL data starts by folding an assumed source distribution through the instrumental response (PSF). The PSF incorporates all of the various physical processes alluded to in $\S 2$ as well as the various event selections which have been imposed on the data. The known exposure and geometric factors (which are specific to a given observation and include, for example, the on/off status of the various modules) are then included. Finally, a background model is also added in. For the present analysis, we do not use an independent estimate of the background. Instead, an estimate of the background (which is predominantly instrumental in origin) was derived directly from the source data by an averaging technique which suppresses point-source signals while preserving the general background structure (Bloemen et al. 1993). Any diffuse emission which may be present within the field of view can be included as part of the background model. The final (modeled) event distribution can then be compared directly with the observed distribution. Subsequent iterations of the source and/or background distributions can then be made until the predicted event distribution agrees with the data.

In practice, the imaging analysis of the COMPTEL data is performed in one of two ways. One technique employs the 
maximum entropy method to derive a source distribution on the sky (Strong et al. 1992; Schönfelder et al. 1993). As presently implemented for COMPTEL data analysis, this algorithm does not provide quantitative error estimates. A more quantitative analysis can be made using a maximum-likelihood technique (de Boer et al. 1992; Schönfelder et al. 1993). This approach, which we have used here, compares the relative probability of a model which contains only background to the probability of a model which contains both the background plus a single point source at the given location (the likelihood ratio). This method provides quantitative information regarding both the source location and the flux together with their associated errors.

As an example of the maximum-likelihood imaging process, Figure 1 shows a maximum likelihood map from viewing period 7.0 for the $0.75-1.0 \mathrm{MeV}$ energy range. The contours represent constant values of the quantity $-2 \ln \lambda$, where $\lambda$ is the likelihood ratio. In a search for single point sources, $-2 \ln \lambda$ has a chi-square distribution with 3 d.o.f. (For instance, a $3 \sigma$ detection corresponds to $-2 \ln \lambda=13.9$ ).) In this case, there is strong evidence for a point source near the location of Cygnus X-1 (marked with a cross). The significance of this detection is $7.7 \sigma(-2 \ln \lambda=58.4)$. A map of the location contours is displayed in Figure 2, showing that the location of Cygnus $\mathrm{X}-1$ is just within the $3 \sigma$ contour for the observed source. The contours reflect only the statistical uncertainties; systematic effects are not included.

\section{RESULTS}

The initial data analysis provided evidence for positive flux measurements at energies up to $2 \mathrm{MeV}$ in both viewing periods, with a negligible number of events at energies above 2 $\mathrm{MeV}$ (McConnell et al. 1993). Here, a finer energy binning was used. In total, six separate energy bins were defined: $0.75-0.85$, $0.85-1.0,1-2,2-5,5-10$, and $10-30 \mathrm{MeV}$. This binning provided three significant data points below $2 \mathrm{MeV}$.

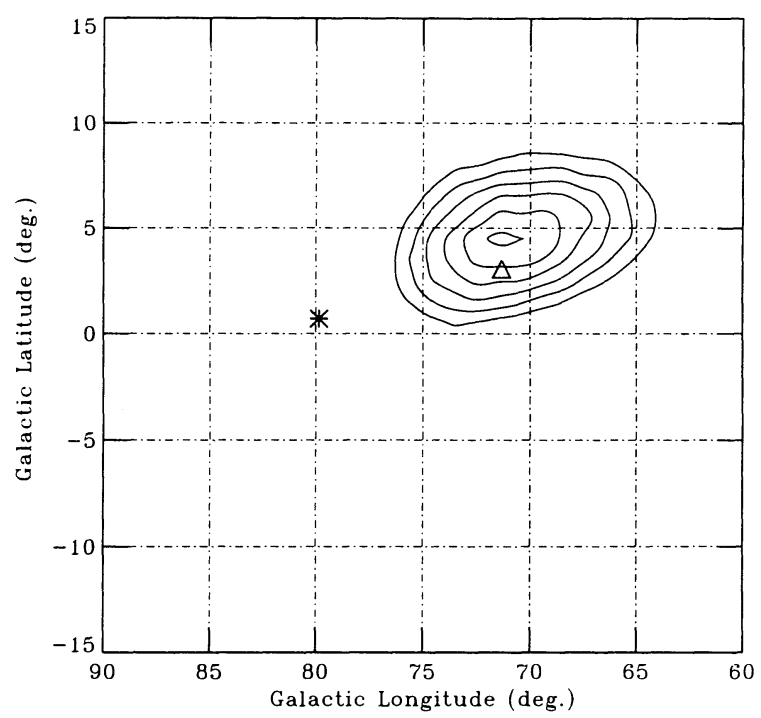

Fig. 1.-Maximum-likelihood map of the Cygnus region compiled using the $0.75-1.0 \mathrm{MeV}$ data from viewing period 7.0. The contours represent constant values of the quantity $-2 \ln \lambda$, where $\lambda$ is the likelihood ratio (see text). The contour levels start at a value of 20 and are incremented by 8 . The response from Cygnus X-1 (denoted by triangle) dominates the image. There is no evidence of any signal in the vicinity of Cygnus X-3 (denoted by asterisk).

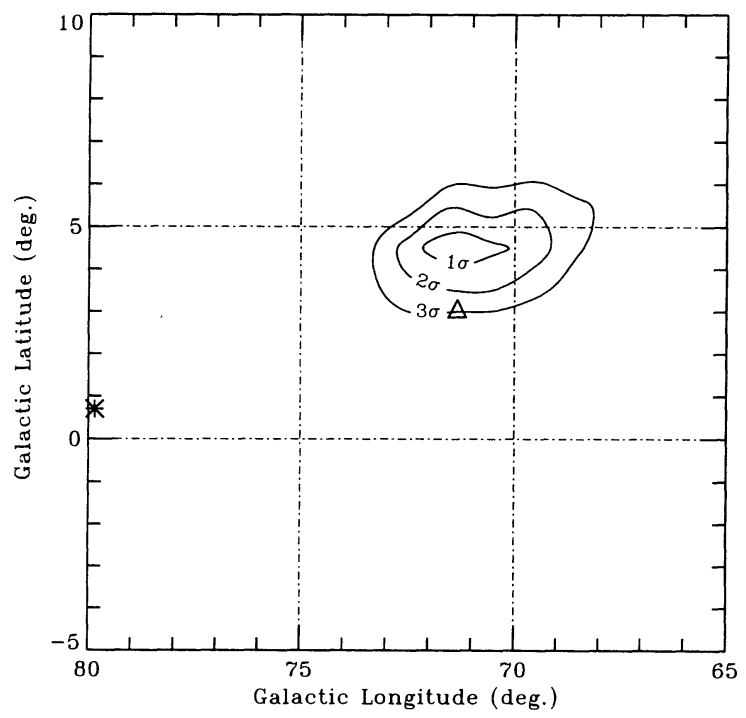

FIG. 2.-A location contour map for the same data as in Fig. 1. The actual location of Cygnus X-1 (denoted by triangle) lies within the $3 \sigma$ contour. The location of Cygnus X-3 is marked by the asterisk. Note that the scale in this figure is slightly larger than that in Fig. 1.

For the low-energy bands (i.e., those below $2 \mathrm{MeV}$ ), simulated PSFs derived from a Wien spectrum have been used in the analysis. Various values of the electron temperature, ranging from 80 to $300 \mathrm{keV}$, have been used to generate these PSFs. Within this range of $k T$ values, the derived source flux is relatively insensitive to the value used in generating the PSF. (That this is the case can be seen in Tables 2 and 3.) Due to the very steep nature of the Wien spectrum near $1 \mathrm{MeV}$, any detectable emission at energies above $\sim 2 \mathrm{MeV}$ must involve a hardening of the spectrum. For this reason, an $E^{-2}$ power law is used in generating PSFs above $2 \mathrm{MeV}$.

The measured flux values (which are given in Tables 2 and 3 for viewing periods 2.0 and 7.0, respectively) show that there is a significant change in the source intensity between the June observation (2.0) and the August observation (7.0). Integrated over the entire $0.75-2.0 \mathrm{MeV}$ energy range, the total flux changed from $3.17( \pm 0.55) \times 10^{-4} \mathrm{~cm}^{-2} \mathrm{~s}^{-1}$ in June to $6.64( \pm 0.74) \times 10^{-4} \mathrm{~cm}^{-2} \mathrm{~s}^{-1}$ in August. This change in flux level (by about a factor of 2) is significant at the $3.8 \sigma$ level. In both cases, contemporaneous OSSE observations (Johnson et al. 1993; Grabelsky et al. 1993) found that the $100 \mathrm{keV}$ flux was consistent with the $\gamma_{2}$ level as defined by Ling et al. (1987).

The COMPTEL spectra from viewing periods 2.0 and 7.0 are shown (along with the contemporaneous OSSE spectra) in Figures 3 and 4, respectively. In both cases, the extrapolation of the OSSE spectrum to the COMPTEL spectrum is a very smooth one with no evidence of any significant spectral hardening.

Given that the measured flux levels in the individual data points are relatively insensitive to the form of the assumed spectrum, we have used the measured flux values to fit the spectrum directly in photon space (i.e., in $d N / d E$ space). For viewing period 2.0 , we obtain a $k T$ value of $192 \pm 27 \mathrm{keV}$ [reduced $\chi^{2}=0.08$ for 1 d.o.f.; $P\left(>\chi^{2}\right)=78 \%$ ]. For viewing period 7.0, we obtain a $k T$ value of $204 \pm 21 \mathrm{keV}$ [reduced $\chi^{2}=3.50$ for 1 d.o.f.; $P\left(>\chi^{2}\right)=6 \%$ ]. We have also cbtained crude estimates for $k T$ from both observations using the ratio of the flux in the $0.75-1.0 \mathrm{MeV}$ interval to that in the $1.0-3.0$ 
No. 2, 1994

OBSERVATIONS OF CYGNUS X-1 BY COMPTEL

TABLE 2

Measured Flux Values: Viewing Period 2.0

\begin{tabular}{|c|c|c|c|c|}
\hline $\begin{array}{l}\text { Energy } \\
(\mathrm{MeV})\end{array}$ & $\begin{array}{c}\text { Response kT } \\
(\mathrm{keV})\end{array}$ & Counts & $\begin{array}{c}\text { Flux } \\
\left(\mathrm{cm}^{-2} \sec ^{-1} \mathrm{MeV}^{-1}\right)\end{array}$ & $\begin{array}{l}\text { Significance } \\
\text { (signa) }\end{array}$ \\
\hline \multirow[t]{5}{*}{$0.75-0.85$} & 100 & $128 \pm 37$ & $9.3( \pm 2.7) \times 10^{-4}$ & 3.5 \\
\hline & 150 & $136 \pm 39$ & $8.9( \pm 2.6) \times 10^{-4}$ & 3.5 \\
\hline & 200 & $150 \pm 45$ & $8.5( \pm 2.6) \times 10^{-4}$ & 3.3 \\
\hline & 250 & $171 \pm 49$ & $9.0( \pm 2.6) \times 10^{-4}$ & 3.5 \\
\hline & 300 & $213 \pm 55$ & $1.0( \pm 0.3) \times 10^{-3}$ & 3.9 \\
\hline \multirow[t]{5}{*}{$0.85-1.0$} & 100 & $229 \pm 61$ & $6.3( \pm 1.7) \times 10^{-4}$ & 3.7 \\
\hline & 150 & $260 \pm 67$ & $6.6( \pm 1.7) \times 10^{-4}$ & 3.9 \\
\hline & 200 & $284 \pm 74$ & $6.6( \pm 1.7) \times 10^{-4}$ & 3.8 \\
\hline & 250 & $360 \pm 81$ & $7.7( \pm 1.7) \times 10^{-4}$ & 4.5 \\
\hline & 300 & $331 \pm 86$ & $6.7( \pm 1.8) \times 10^{-4}$ & 3.9 \\
\hline \multirow[t]{5}{*}{$1.0-2.0$} & 100 & $444 \pm 145$ & $1.4( \pm 0.5) \times 10^{-4}$ & 3.1 \\
\hline & 150 & $477 \pm 15: 3$ & $1.4( \pm 0.5) \times 10^{-4}$ & 3.1 \\
\hline & 200 & $488 \pm 162$ & $1.3( \pm 0.4) \times 10^{-4}$ & 3.0 \\
\hline & 250 & $493 \pm 170$ & $1.2( \pm 0.4) \times 10^{-4}$ & 2.9 \\
\hline & 300 & $497 \pm 177$ & $1.2( \pm 0.4) \times 10^{-4}$ & 2.8 \\
\hline $2.0-5.0$ & $E^{-2} \mathrm{PL}$ & $197 \pm 151$ & $1.2( \pm 0.9) \times 10^{-.5}$ & 1.3 \\
\hline $5.0-10.0$ & $E^{-2} \mathrm{PL}$ & $-170 \pm 56$ & $<4.1 \times 10^{-6}(2 \sigma)$ & \\
\hline $10.0-30.0$ & $E^{-2} \mathrm{PL}$ & $-16 \pm 2: 3$ & $<3.7 \times 10^{-7}(2 \sigma)$ & \\
\hline
\end{tabular}

$\mathrm{MeV}$ interval. The measured flux ratios can be compared with that expected for various values of the electron temperature. For viewing period 2.0 , we estimate $k T$ to be $\sim 170 \mathrm{keV}$. For viewing period 7.0, we estimate $k T$ to be $\sim 200 \mathrm{keV}$. These numbers are consistent with the results of the direct spectral fits given above.

\section{DISCUSSION}

The temperatures which we derive for a Wien-type spectrum differ considerably from typical temperatures measured at hard $\mathrm{X}$-ray energies. In the past, the hard X-ray data (at energies below $\sim 300 \mathrm{keV}$ ) have normally been fitted using a single-

TABLE 3

Measured Flux Values: Viewing Period 7.0

\begin{tabular}{|c|c|c|c|c|}
\hline $\begin{array}{l}\text { Energy } \\
(\mathrm{MeV})\end{array}$ & $\begin{array}{c}\text { Response kT } \\
(\mathrm{keV})\end{array}$ & Counts & $\begin{array}{c}\text { Flux } \\
\left(\mathrm{cm}^{-2} \mathrm{sec}^{-1} \mathrm{MeV}^{-1}\right)\end{array}$ & $\begin{array}{l}\text { Significance } \\
\text { (sigma) }\end{array}$ \\
\hline \multirow[t]{5}{*}{$0.75-0.85$} & 100 & $196 \pm 31$ & $2.4( \pm 0.4) \times 10^{-3}$ & 6.3 \\
\hline & 150 & $182 \pm 32$ & $2.0( \pm 0.4) \times 10^{-3}$ & 5.7 \\
\hline & 200 & $217 \pm 36$ & $2.1( \pm 0.3) \times 10^{-3}$ & 6.1 \\
\hline & 250 & $219 \pm 38$ & $2.0( \pm 0.3) \times 10^{-3}$ & 5.7 \\
\hline & 300 & $237 \pm 43$ & $2.0( \pm 0.4) \times 10^{-3}$ & 5.6 \\
\hline \multirow[t]{5}{*}{$0.85-1.0$} & 100 & $194 \pm 49$ & $8.3( \pm 2.1) \times 10^{-4}$ & 4.0 \\
\hline & 150 & $207 \pm 54$ & $8.4( \pm 2.2) \times 10^{-4}$ & 3.9 \\
\hline & 200 & $220 \pm 59$ & $8.2( \pm 2.2) \times 10^{-4}$ & 3.8 \\
\hline & 250 & $239 \pm 63$ & $8.1( \pm 2.2) \times 10^{-4}$ & 3.8 \\
\hline & 300 & $255 \pm 67$ & $8.4( \pm 2.2) \times 10^{-4}$ & 3.8 \\
\hline \multirow[t]{5}{*}{$1.0-2.0$} & 100 & $657 \pm 119$ & $3.2( \pm 0.6) \times 10^{-4}$ & 5.5 \\
\hline & 150 & $744 \pm 126$ & $3.4( \pm 0.6) \times 10^{-4}$ & 5.9 \\
\hline & 200 & $822 \pm 133$ & $3.4( \pm 0.6) \times 10^{-4}$ & 6.1 \\
\hline & 250 & $877 \pm 139$ & $3.4( \pm 0.5) \times 10^{-4}$ & 6.3 \\
\hline & 300 & $947 \pm 145$ & $3.6( \pm 0.6) \times 10^{-4}$ & 6.5 \\
\hline $2.0-5.0$ & $E^{-2} \mathrm{PL}$ & $241 \pm 127$ & $2.3( \pm 1.2) \times 10^{-5}$ & 1.3 \\
\hline $5.0-10.0$ & $E^{-2} \mathrm{PL}$ & $-101 \pm 48$ & $<5.8 \times 10^{-6}(2 \sigma)$ & \\
\hline $10.0-30.0$ & $E^{-2} \mathrm{PL}$ & $-77 \pm 17$ & $<4.9 \times 10^{-7}(2 \sigma)$ & \\
\hline
\end{tabular}




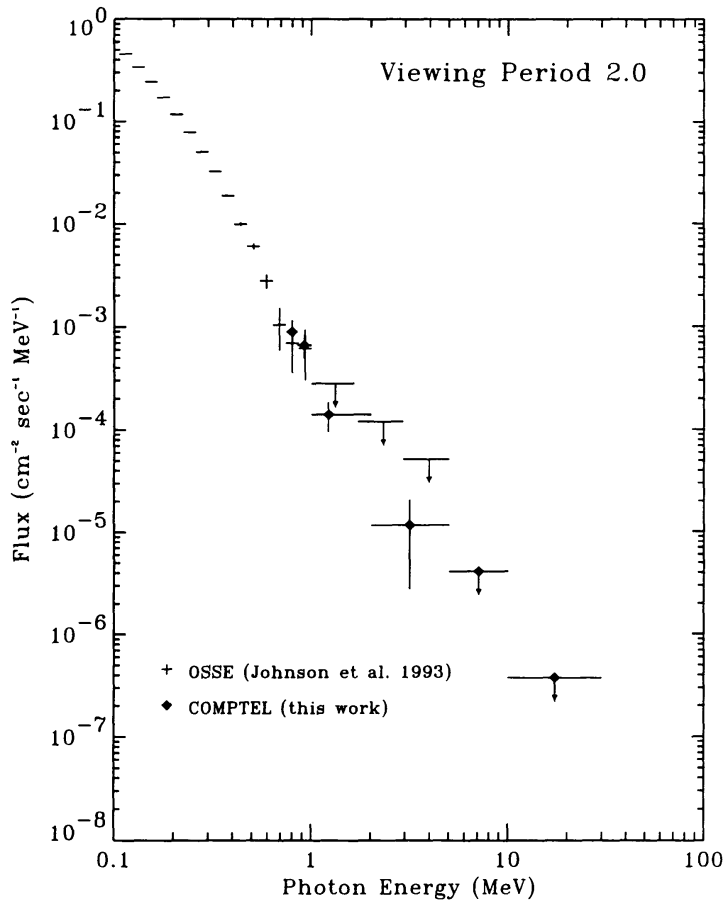

Fig. 3.-The COMPTEL spectral data points from viewing period 2.0 (1991 May 30-Jun 8) plotted along with the OSSE spectrum from the same time period (Johnson et al. 1993). The extrapolation of the OSSE spectrum through the COMPTEL data points is a very smooth one, with no significant evidence for a hardening of the spectrum.

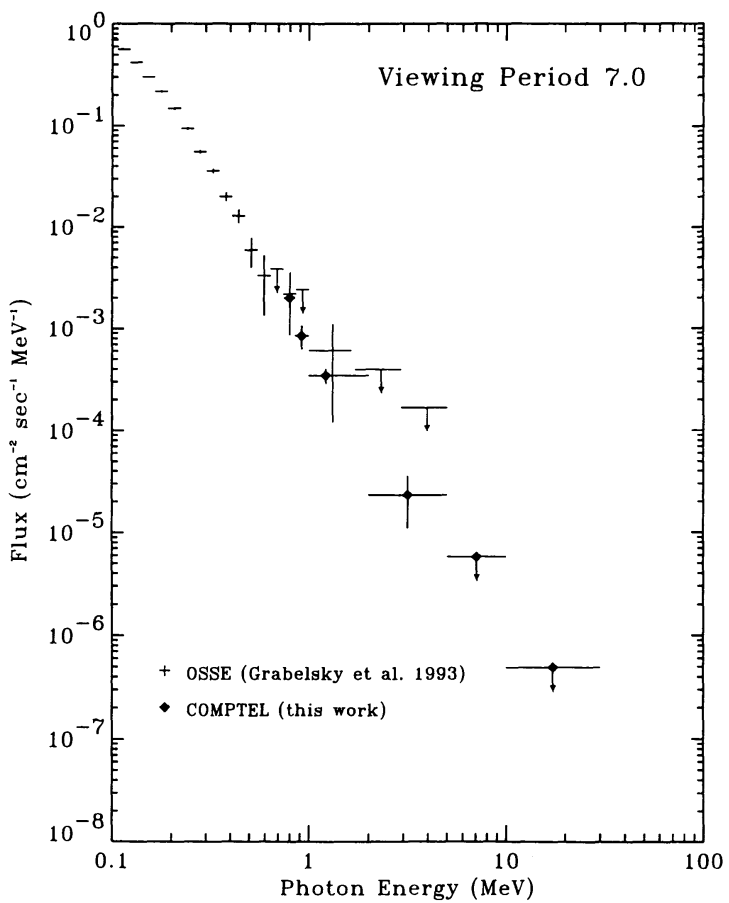

FIG. 4.-The COMPTEL spectral data points from viewing period 7.0 (1991 August 8-15) plotted along with the OSSE spectrum from the same time period (Grabelsky et al. 1993). As in Fig. 3, the extrapolation of the OSSE spectrum through the COMPTEL data points is a very smooth one, with no significant evidence for a hardening of the spectrum. Near $1 \mathrm{MeV}$, the flux level indicated by these data is about a factor of 2 higher than the flux level in viewing period 2.0 (Fig. 3). temperature Sunyaev-Titarchuk Comptonization model; typical electron temperatures derived from these fits are in the 40-80 keV range. Observations extending up to $1 \mathrm{MeV}$ (e.g., Nolan \& Matteson 1983; Grebenev et al. 1993; Grabelsky et al. 1993) suggest that a single-temperature Comptonization model does not provide an adequate fit at energies above $\sim 300 \mathrm{keV}$. Although a two-temperature Comptonization model tends to give a better fit, these results are often used to suggest that a more complete modeling of the Comptonization process may be required (e.g., Grebenev et al. 1993; Grabelsky et al. 1993). Such a study would seek to overcome the limitations of the analytical approach (Pozdnyakov, Sobol, \& Sunyaev 1983). For instance, Grebenev et al. (1993) have found that a Monte Carlo modeling of the Comptonization spectrum provides a much better fit to the broad-band data from Granat. So the discrepancy which we find between our results and the hard X-ray results may be due to the inadequacy of the model used in the analysis of hard X-ray data.

Another plausible explanation of our results is presented by Haardt et al. (1993). They suggest a model that consists of a hot (optically thin) accretion disk corona surrounding a much cooler (optically thick) accretion disk. In addition to the spectral component which we observe directly from the corona, there is a backscattered component from the hard X-rays scattering off the cooler accretion disk. A fit of this model to EXOSAT data suggests coronal electron temperatures in the range of $130-200 \mathrm{keV}$ and optical depths less than unity $(\tau \sim 0.3)$. In the COMPTEL energy range, the proposed backscattered component is negligible, and one would conclude that, in the context of this model, the COMPTEL data represent a direct measure of the coronal temperature. The temperatures derived in our analysis $(\sim 190-220 \mathrm{keV})$ are similar to those suggested for the coronal temperature as derived from EXOSAT data (Haardt et al. 1991).

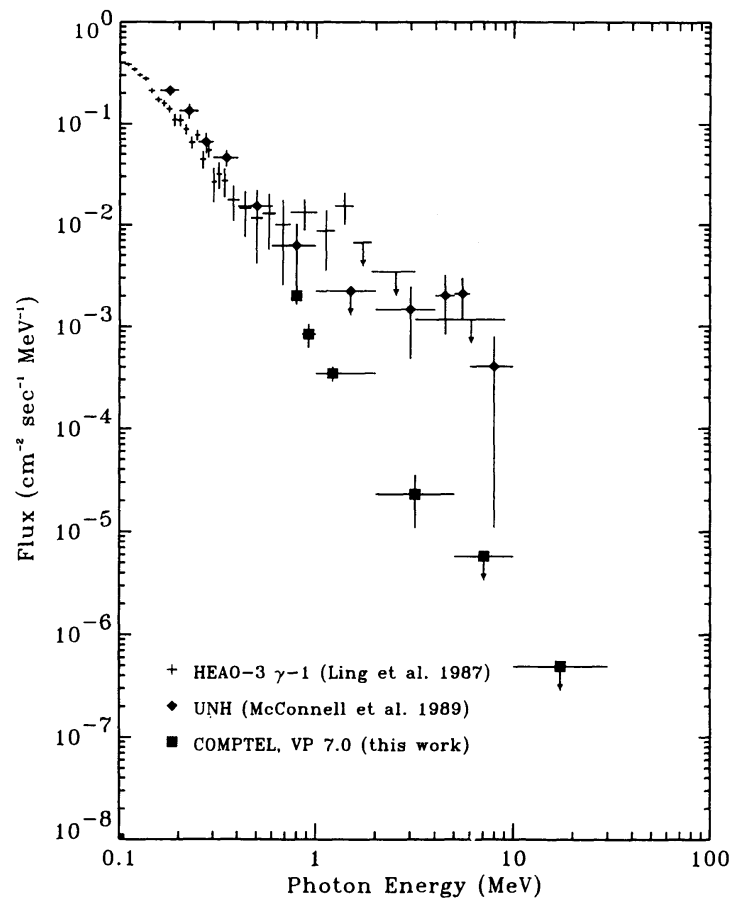

Fig. 5.-A comparison between the COMPTEL data from viewing period 7.0 with selected data from previous experiments. The COMPTEL data points near $1 \mathrm{MeV}$ are more than an order of magnitude below any of the positive flux measurements which have been previously reported. 
One of our goals in studying the emission from Cygnus X-1 was to look for any excess emission beyond that which is expected based on an extrapolation of the hard X-ray spectrum. From Figures 3 and 4, we see no evidence for any such excess in both viewing period 2.0 and in viewing period 7.0. Figure 5 shows the COMPTEL data from viewing period 7.0 relative to some of the previous measurements near $1 \mathrm{MeV}$. The most significant of the positive results is that of $H E A O 3$ (Ling et al. 1987). At $1 \mathrm{MeV}$, the COMPTEL data are more than an order of magnitude below the HEAO 3 data. A similar conclusion can be drawn by comparison with the balloon data of McConnell et al. (1989). However, the UCR Compton telescope upper limits (White et al. 1980) are in agreement with the COMPTEL results.

The possibility that some type of pair-plasma process or some type of nonthermal process may be taking place within the Cygnus X-1 accretion flow at the time of the COMPTEL observations is not supported by the present measurements. On the basis of the earlier data, there is clearly a time variable nature to the "MeV excess." Unfortunately, most of these data at energies greater than $1 \mathrm{MeV}$ were of a rather low statistical significance. For example, the flux reported by McConnell et al. (1989) has a significance of $2.9 \sigma$ for the emission above 1 $\mathrm{MeV}$. A confirmation of this emission would therefore prove extremely valuable. Further confirmation of the HEAO 3 results would also be of great interest, especially given that more sensitive experiments (e.g., OSSE and COMPTEL) have so far failed to provide a confirmation of those data.

From an observational perspective, it would be useful to develop a means of identifying black hole binary systems which is independent of any dynamical data. Although no definitive criteria have been established, it is generally believed that the high-energy signatures of black hole candidates include both a variable power-law $\gamma$-ray tail (with a photon number index $<2$ and an exponential cutoff above a few hundred $\mathrm{keV}$ ) as well as transient $\gamma$-ray bumps above $\sim 400$ $\mathrm{keV}$ (Liang 1992). Cygnus X-1 exhibits both of these characteristics. It is also becoming increasingly clear (e.g., Done et al. 1992; Owens \& McConnell 1992; Haardt et al. 1993) that certain soft X-ray features should also be incorporated into this standard model. In conjunction with the results of Haardt et al. (1993), who used EXOSAT data to infer a high coronal temperature, the present results may support the need for a backscattered component to more fully explain the X-ray and $\gamma$-ray spectrum of Cygnus X-1.

\section{SUMMARY}

The present COMPTEL results represent a high-sensitivity measurement of the Cygnus X-1 spectrum to energies above 1 $\mathrm{MeV}$. Although there is evidence for significant variability in the flux near $1 \mathrm{MeV}$, these data show no evidence for any excess emission as has sometimes been reported in the past. The present results provide supporting evidence for the model proposed by Haardt et al. (1993), which includes a backscattered component in the hard X-ray emission and suggests a much higher electron temperature than previously considered. Further insight may be expected as we continue our analysis of the COMPTEL data. This will include several weeks of data collected during phase 2 of the CGRO mission.

We would like to thank M. Leising and J. Kurfess for providing the OSSE spectra which are included here.
Aharonian, F. A., \& Sunyaev, R. A. 1984, MNRAS, 210, 257

Baker, R. E., et al. 1973, Nature Phys. Sci., 245, 18

Balucinska, M., \& Hasinger, G. 1991, A\&A, 241, 439

Barr, P., White, N. E., \& Page, C. G. 1985, MNRAS, 216, 65p

Bassani, L., et al. 1989, ApJ, 343, 313

Bednarek, W., et al. 1990, A\&A, 236, 175

Bloemen, H., et al. 1993, ApJS, in press

Dahlbacka, G. H., Chapline, G. F., \& Weaver, T. A. 1974, Nature, 250, 36

de Boer, H., et al. 1992, in Proc. 4th Internat. Workshop on Data Analysis in Astronomy, (Erice, Italy), ed. V. Di Gesù et al. (New York: Plenum)

Dermer, C., \& Liang, E. P. 1989, ApJ, 339, 512

Dolan, J. F. 1992, ApJ, 384, 249

Dolan, J. F., \& Tapia, S. 1989, ApJ, 344, 830

Done, C., Mulchaey, J. S., Mushotzky, R. F., \& Arnaud, K. A. 1992, ApJ, 395, 275

Eilek, J. 1980, ApJ, 236, 664

Eilek, J., \& Kafatos, M. 1983, ApJ, 271, 804

Giovanelli, F., Karakula, S., \& Tkaczyk, W. 1982, A\&A, 107, 376

Gies, D. R., \& Bolton, C. T. 1986, ApJ, 304, 371

Grabelsky, D. A., et al. 1993, in Proc. Compton Symp. (St. Louis), ed. M. Friedlander et al. (New York: AIP), 345

Grebenev, S., et al. 1993, A\&AS, 97, 281

Guessom, N. 1989, ApJ, 345, 363

Guessom, N., \& Dermer, C. D. 1988, in AIP Conf. Proc. 107, Nuclear Spectroscopy of Astrophysical Sources, ed. N. Gehrels \& G. H. Share (New York: AIP), 332

Haardt, F., Done, C., Matt, G., \& Fabian, A. C. 1993, ApJ, 411, L95

Higdon, J. C., \& Lingenfelter, R. E. 1977, ApJ, 215, L53

Johnson, W. N., et al. 1993, A\&AS, 97, 21

Kolykhalov, P. I., \& Sunyaev, R. A. 1979, Soviet Astron., 23, 189

Leising, M. D., Strickman, M. S., Johnson, W. N., Kurfess, J. D., \& Grove, J. E. 1993, IAU Circ., No. 5823

\section{REFERENCES}

Liang, E. P. 1990, A\&A, 227, 447 3137,173

Liang, E. P., \& Dermer, C. D. 1988, ApJ, 325, L39

Lightman, A. P., \& White, T. R. 1988, ApJ, 335, 57

Ling, J. C., Mahoney, W. A., Wheaton, W. A., Jacobsen, A. S., \& Kaluzienski, L. 1987, ApJ, 321, L117

Mandrou, P., et al. 1978, ApJ, 219, 288

Matt, G., Perola, G. C., \& Piro, L. 1991, A\&A, 247, 25

McConnell, M. L., et al. 1989, ApJ, 343, 317

. 1993, in Proc. Compton Symp. (St. Louis), ed. M. Friedlander et al. (New York: AIP), 335

Nolan, P., \& Matteson, J. L. 1983, ApJ, 265, 389

Owens, A., \& McConnell, M. 1992, Comm. Astrophys., 16, 205

Pounds, K. A., Nandra, K., Stewart, G. C., George, I. M., \& Fabian, A. C. 1990 Nature, 344, 132

Pozdnyakov, L. A., Sobol, I. M., \& Sunyaev, R. A. 1983, Ap\&SS, 2, 263

Ross, R. R., \& Fabian, A. C. 1993, MNRAS, 261, 74

Ross, R. R., Fabian, A. C., \& Mineshige, S. 1992, MNRAS, 258, 189

Schönfelder, V., \& Lichti, G. 1974, ApJ, 192, L1

Schönfelder, V., et al. 1993, ApJS, 86, 629

Shapiro, S. L., Lightman, A. P., \& Eardley, D. M. 1976, ApJ, 204, 187

Strong, A. W., et al. 1992, in Proc. 4th Internat. Workshop on Data Analysis in Astronomy, (Erice, Italy), ed. V. Di Gesù et al. (New York: Plenum), 251

Sunyaev, R. A., \& Titarchuk, L. G. 1980, A\&A, 86, 121

Varendorff, M., von Ballmoos, P., Graser, U., \& Schönfelder, V. 1990, Proc. 21st International Cosmic Ray Conf. (Adelaide), 1,200

White, R. S., Dayton, B., Gibbons, R., Long, J. L., Znrosso, E. M., \& Zych, A. D. 1980 , Nature, 284,608

White, N. E., Fabian, A. C., \& Mushotzky, R. F. 1984, A\&A, 133, L9

Winkler, C., et al. 1992, A\&A, 255, L9

Note added in proof.-In our discussion of time variability of the "MeV excess," we overlooked the important contribution of Harris et al. (ApJ, 416, 601 [1993]), which provides constraints on variability based on SMM-GRS observations during the period 1981-1989. In particular, these data indicate that any emission at the level reported by McConnell et al. (1989) could not have lasted more than 1 day. 